Supporting Information for:

\title{
Remote Asymmetric Induction About a Crowded Aromatic Core
}

\author{
A. J. Lampkins, O. Abdul-Rahim, R. K. Castellano* \\ Department of Chemistry, University of Florida, Gainesville, FL 32611
}

\section{Table of Contents}

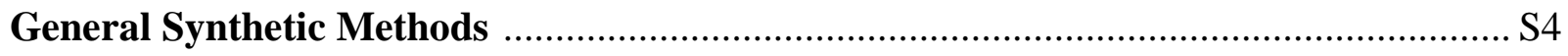

Synthesis and Characterization of Compounds ……................................................. S4

2,4,6-Trimethoxybenzene-1,3,5-tricarbaldehyde (1) .................................................. S4

(anti, syn)-(2,4,6-Trimethoxybenzene-1,3,5-triyl)tris(phenylmethanol) (3a) .................. S4

2,4,6-Triethylbenzene-1,3,5-tricarbaldehyde (4) ..................................................... S5

(anti,syn)-1,1',1"-(2,4,6-Triethylbenzene-1,3,5-triyl)triethanol (6a) .............................. S5

(syn,syn)-1,1',1"-(2,4,6-Triethylbenzene-1,3,5-triyl)triethanol (6b) .............................. S6

1,1',1"-(2,4,6-Trimethoxybenzene-1,3,5-triyl)triethanone (7) ....................................... S6

1,1',1"-(2,4,6-Triethylbenzene-1,3,5-triyl)triethanone (8) ............................................ S6

meso-3-(1-Hydroxyethyl)-5-(1-hydroxyethyl)-2,4,6-trimethoxybenzaldehyde (meso-9)

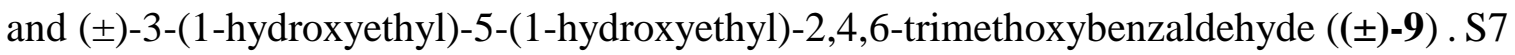

${ }^{1} \mathrm{H}(300 \mathrm{MHz})$ and ${ }^{13} \mathrm{C}(75 \mathrm{MHz})$ NMR of New Compounds ........................................ S8

${ }^{1} \mathrm{H}$ NMR spectrum of 2,4,6-trimethoxybenzene-1,3,5-tricarbaldehyde (1) .................... S8

${ }^{13} \mathrm{C}$ NMR spectrum of 2,4,6-trimethoxybenzene-1,3,5-tricarbaldehyde (1) .................... S9

${ }^{1} \mathrm{H}$ NMR spectrum of (anti, syn)-(2,4,6-trimethoxybenzene-1,3,5-triyl)tris-

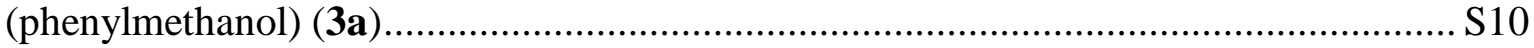

${ }^{13}$ C NMR spectrum of (anti, syn)-(2,4,6-trimethoxybenzene-1,3,5-triyl)tris-

(phenylmethanol) (3a) 
${ }^{1} \mathrm{H}$ NMR spectrum of 2,4,6-triethylbenzene-1,3,5-tricarbaldehyde (4) $\mathrm{S} 12$

${ }^{13} \mathrm{C}$ NMR spectrum of 2,4,6-triethylbenzene-1,3,5-tricarbaldehyde (4) S13

${ }^{1} \mathrm{H}$ NMR spectrum of (anti,syn)-1,1',1"-(2,4,6-trimethoxybenzene-1,3,5-triyl)triethanol (5a) S14

${ }^{13} \mathrm{C}$ NMR spectrum of (anti,syn)-1,1',1"-(2,4,6-trimethoxybenzene-1,3,5-triyl)-

triethanol (5a) S15

${ }^{1} \mathrm{H}$ NMR spectrum of (syn,syn)-1,1',1"-(2,4,6-trimethoxybenzene-1,3,5-triyl)-

triethanol (5b) S16

${ }^{13} \mathrm{C}$ NMR spectrum of (syn,syn)-1,1',1"-(2,4,6-trimethoxybenzene-1,3,5-triyl)-

triethanol (5b)

${ }^{1} \mathrm{H}$ NMR spectrum of (anti,syn)-1,1',1"-(2,4,6-triethylbenzene-1,3,5-triyl)-

triethanol (6a)

${ }^{13} \mathrm{C}$ NMR spectrum of (anti,syn)-1,1',1"-(2,4,6-triethylbenzene-1,3,5-triyl)-

triethanol (6a)

${ }^{1} \mathrm{H}$ NMR spectrum of (syn,syn)-1,1',1"-(2,4,6-triethylbenzene-1,3,5-triyl)-

triethanol (6b) S20

${ }^{13} \mathrm{C}$ NMR spectrum of (syn,syn)-1,1',1"-(2,4,6-triethylbenzene-1,3,5-triyl)-

triethanol $(\mathbf{6 b})$ S21

${ }^{1} \mathrm{H}$ NMR spectrum of 1,1',1"-(2,4,6-trimethoxybenzene-1,3,5-triyl)triethanone (7)....... S22

${ }^{13} \mathrm{C}$ NMR spectrum of 1,1',1"-(2,4,6-trimethoxybenzene-1,3,5-triyl)triethanone (7) ...... S23

${ }^{1}$ H NMR spectrum of 1,1',1"-(2,4,6-triethylbenzene-1,3,5-triyl)triethanone (8) ............ S24

${ }^{13} \mathrm{C}$ NMR spectrum of 1,1',1"-(2,4,6-triethylbenzene-1,3,5-triyl)triethanone (8) .......... S25 
${ }^{1} \mathrm{H}$ NMR spectrum of meso-3-(1-hydroxyethyl)-5-(1-hydroxyethyl)-2,4,6-

trimethoxybenzaldehyde (meso-9) and ( \pm )-3-(1-hydroxyethyl)-5-(1-hydroxyethyl)-2,4,6trimethoxybenzaldehyde $(( \pm)-9)$ S26

${ }^{13} \mathrm{C}$ NMR spectrum of meso-3-(1-hydroxyethyl)-5-(1-hydroxyethyl)-2,4,6-

trimethoxybenzaldehyde (meso-9) and ( \pm )-3-(1-hydroxyethyl)-5-(1-hydroxyethyl)-2,4,6-

trimethoxybenzaldehyde ((士)-9) 


\section{General Synthetic Methods}

Reagents and solvents were purchased from commercial sources and used without further purification unless otherwise specified. THF, ether, $\mathrm{CH}_{2} \mathrm{Cl}_{2}$, and DMF were degassed in $20 \mathrm{~L}$ drums and passed through two sequential purification columns (activated alumina; molecular sieves for DMF) under a positive argon atmosphere. Thin layer chromatography (TLC) was performed on aluminum sheets with visualization with UV light or staining. Melting points (m.p.) were determined on a standard melting point apparatus and are uncorrected. ${ }^{1} \mathrm{H}(300)$ and ${ }^{13} \mathrm{C}$ NMR (75 MHz) spectra were recorded on a Varian Mercury 300 spectrometer. Chemical shifts $(\delta)$ are given in parts per million ( $\mathrm{ppm})$ relative to TMS and referenced to residual protonated solvent $\left(\mathrm{CHCl}_{3}: \delta_{\mathrm{H}} 7.27 \mathrm{ppm}, \delta_{\mathrm{C}} 77.00 \mathrm{ppm}\right)$. Abbreviations used are s (singlet), $\mathrm{d}$ (doublet), $\mathrm{t}$ (triplet), q (quartet), quin (quintet), b (broad), and m (multiplet). MS spectra (HRMS and LRMS) were recorded on a Finnigan MAT95Q Hybrid Sector spectrometer.

\section{Synthesis and Characterization of Compounds}<smiles>COc1c(C=O)c(OC)c(C=O)c(OC)c1C=O</smiles>

2,4,6-Trimethoxybenzene-1,3,5-tricarbaldehyde (1). This compound was prepared according to the published procedure. ${ }^{1}{ }^{1} \mathrm{H}$ NMR $\left(\mathrm{CDCl}_{3}\right) \delta 4.03(\mathrm{~s}, 9 \mathrm{H}), 10.35(\mathrm{~s}, 3 \mathrm{H}) .{ }^{13} \mathrm{C} \mathrm{NMR}\left(\mathrm{CDCl}_{3}\right)$ $\delta 65.6,120.0,169.7,187.1$.<smiles>COc1c(C(O)c2ccccc2)c(OC)c([C@H](O)c2ccccc2)c(OC)c1[C@H](O)c1ccccc1</smiles>

(anti,syn)-(2,4,6-Trimethoxybenzene-1,3,5-triyl)tris(phenylmethanol) (3a). To a stirring solution of bromobenzene $(0.31 \mathrm{~mL}, 3.0 \mathrm{mmol})$ in dry THF $(15 \mathrm{~mL})$ at $-78^{\circ} \mathrm{C}$ under a blanket of argon, was slowly added a solution of $n$-buLi $(2.5 \mathrm{M}$ in hexanes, $1.2 \mathrm{~mL}, 3.0 \mathrm{mmol})$ and the resulting mixture was allowed to stir for $1 \mathrm{~h}$. A solution of $\mathbf{1}^{1}(0.15 \mathrm{~g}, 0.60 \mathrm{mmol}$, dissolved in 5 $\mathrm{mL}$ dry THF) was then added to the stirring solution via syringe, and the reaction mixture was maintained at $-78{ }^{\circ} \mathrm{C}$ for an additional hour, and then allowed to gradually warm to room temperature over $2 \mathrm{~h}$. The reaction was then quenched by dropwise addition of dilute $\mathrm{HCl}$, and 
extracted with EtOAc. The combined organic extracts were dried over $\mathrm{MgSO}_{4}$, filtered, and concentrated to a crude residue $\left({ }^{1} \mathrm{H}\right.$ NMR analysis of this crude residue showed no signs of epimer 3b), which was purified via flash chromatography (3:2 hexanes/EtOAc) to afford 3a $(0.19 \mathrm{~g}, 67 \%)$ as a colorless solid. All spectral data matches known compound. ${ }^{2}{ }^{1} \mathrm{H}$ NMR $\left(\mathrm{C}_{6} \mathrm{D}_{6}\right) \delta 2.92(\mathrm{~s}, 3 \mathrm{H}), 2.95(\mathrm{~s}, 6 \mathrm{H}), 3.60(\mathrm{bs}, 2 \mathrm{H}), 4.06(\mathrm{bs}, 1 \mathrm{H}), 6.15(\mathrm{bs}, 2 \mathrm{H}), 6.21(\mathrm{bs}, 1 \mathrm{H})$, 6.97-7.02 (m, 3H), 7.06-7.13 (m, 6H), 7.40 (d, J=7.5 Hz, 4H), 7.49 (d, J=7.2 Hz, 2H). ${ }^{13} \mathrm{C}$ NMR $\left(\mathrm{C}_{6} \mathrm{D}_{6}\right) \delta 62.7,68.4,68.5,125.2,125.5,126.6,127.4,127.7,128.2,129.0,129.2,145.3$, 145.5, 157.9.

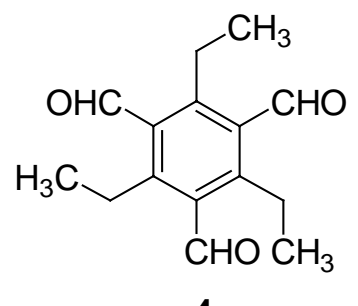

4

2,4,6-Triethylbenzene-1,3,5-tricarbaldehyde (4). PCC (8.55 g, $39.7 \mathrm{mmol})$ was added to a stirring mixture of (2,4,6-triethylbenzene-1,3,5-triyl)methanol ${ }^{3}$ (2.00 g, $\left.7.94 \mathrm{mmol}\right)$ and dry $\mathrm{CH}_{2} \mathrm{Cl}_{2}(150 \mathrm{~mL})$ and the resulting suspension was allowed to stir at room temperature, under argon, overnight. It was then diluted with $\mathrm{CH}_{2} \mathrm{Cl}_{2}$, and suction filtered to remove all solid material. The filtrate was concentrated and purified using column chromatography (6:1 hexanes/EtOAc eluent system) to afford $4(1.17 \mathrm{~g}, 60 \%)$ as a colorless solid: IR (film): $v_{\max }$ 2976, 1696, $1553 \mathrm{~cm}^{-1} ;{ }^{1} \mathrm{H} \mathrm{NMR}\left(\mathrm{CDCl}_{3}\right) \delta 1.25(\mathrm{t}, J=7.5 \mathrm{~Hz}, 9 \mathrm{H}), 2.99$ (q, $\left.J=7.5 \mathrm{~Hz}, 6 \mathrm{H}\right)$, $10.60(\mathrm{~s}, 3 \mathrm{H}) .{ }^{13} \mathrm{C} \mathrm{NMR}\left(\mathrm{CDCl}_{3}\right) \delta 16.5,22.5,134.1,149.2,194.1$. HRMS (ESI) calcd for $\mathrm{C}_{15} \mathrm{H}_{18} \mathrm{O}_{3}(\mathrm{M}+\mathrm{Na})^{+} 269.1148$, found 269.1160 .

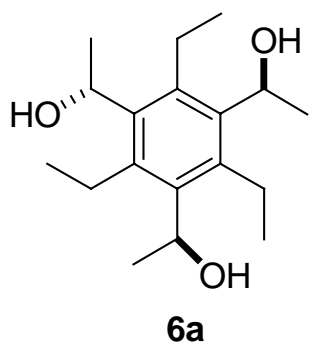

(anti,syn)-1,1',1"-(2,4,6-Triethylbenzene-1,3,5-triyl)triethanol (6a). Method A: This compound was synthesized from methyllithium $(1.6 \mathrm{M}$ in ether, $2.5 \mathrm{~mL}, 4.1 \mathrm{mmol})$ and $4(0.20 \mathrm{~g}$, $0.81 \mathrm{mmol}$ ) according to the same procedure reported for 5a Method A (see manuscript text). Purification of the crude reaction mixture was performed using column chromatography (1:1 hexanes/EtOAc) to afford $6 \mathbf{a}(0.16 \mathrm{~g}, 65 \%)$ and $\mathbf{6 b}(0.049 \mathrm{~g}, 21 \%)$ as colorless solids.

Method B: This compound was synthesized from methyllithium $(1.6 \mathrm{M}$ in ether, $2.5 \mathrm{~mL}$, $4.1 \mathrm{mmol})$, HMPA $(0.71 \mathrm{~mL}, 4.1 \mathrm{mmol})$ and $4(0.20 \mathrm{~g}, 0.81 \mathrm{mmol})$ according to the same procedure reported for $\mathbf{5 b}$. Purification of the crude reaction mixture was performed using column chromatography $(1: 1$ hexanes/EtOAc) to afford $\mathbf{6 a}(0.18 \mathrm{~g}, 75 \%)$ and $\mathbf{6 b}(0.058 \mathrm{~g}, 24 \%)$.

Method $C$ : This compound was synthesized from $\mathrm{LiAlH}_{4}(0.066 \mathrm{~g}, 1.7 \mathrm{mmol})$ and 8 $(0.050 \mathrm{~g}, 0.17 \mathrm{mmol})$ according to the same procedure reported for 5a Method $B$. The crude 
product was purified using column chromatography (1:1 hexanes/EtOAc) to afford $\mathbf{6 a}(0.031 \mathrm{~g}$, $61 \%)$ and $6 \mathbf{b}(0.011 \mathrm{~g}, 21 \%): \mathrm{IR}$ (film): $v_{\max } 3365,2971,1124,1057 \mathrm{~cm}^{-1} ;{ }^{1} \mathrm{H} \mathrm{NMR}\left(\mathrm{CDCl}_{3}\right) \delta$ $1.20(\mathrm{t}, J=7.2 \mathrm{~Hz}, 9 \mathrm{H}), 1.62(\mathrm{~m}, 9 \mathrm{H}), 1.93(\mathrm{bs}, 3 \mathrm{H}), 3.09(\mathrm{bs}, 6 \mathrm{H}), 5.57(\mathrm{~m}, 3 \mathrm{H}) .{ }^{13} \mathrm{C} \mathrm{NMR}$ $\left(\mathrm{CDCl}_{3}\right) \delta 17.3,17.4,22.6,23.6,23.7,66.6,66.7,140.0,141.2$. HRMS (ESI) calcd for $\mathrm{C}_{18} \mathrm{H}_{30} \mathrm{O}_{3}$ $(\mathrm{M}+\mathrm{Na})^{+}$317.2087, found 317.2101.<smiles>CCc1c(C(C)O)c(CC)c(C(C)O)c(CC)c1C(C)O</smiles>

(syn, syn)-1,1',1"-(2,4,6-Triethylbenzene-1,3,5-triyl)triethanol (6b). IR (nujol): $v_{\max } 3326$, 2922, 1457, 1374, 1124, $1059 \mathrm{~cm}^{-1} ;{ }^{1} \mathrm{H} \mathrm{NMR}\left(\mathrm{CDCl}_{3}\right) \delta 1.20(\mathrm{t}, J=7.2 \mathrm{~Hz}, 9 \mathrm{H}), 1.60(\mathrm{~d}, J=5.1$ $\mathrm{Hz}, 9 \mathrm{H}), 2.05(\mathrm{bs}, 3 \mathrm{H}), 2.92(\mathrm{bs}, 3 \mathrm{H}), 3.12(\mathrm{~m}, 3 \mathrm{H}), 5.54(\mathrm{~m}, 3 \mathrm{H}) .{ }^{13} \mathrm{C} \mathrm{NMR}\left(\mathrm{CDCl}_{3}\right) \delta 17.4$, 22.6, 23.5, 66.7, 140.0, 141.0. HRMS (ESI) calcd for $\mathrm{C}_{18} \mathrm{H}_{30} \mathrm{O}_{3}(\mathrm{M}+\mathrm{Na})^{+}$317.2087, found 317.2104 .<smiles>COc1c(C(C)=O)c(OC)c(C(C)=O)c(OC)c1C(C)=O</smiles>

1,1',1'-(2,4,6-Trimethoxybenzene-1,3,5-triyl)triethanone (7). To a stirring mixture of 5a and 5b (1.19 g, $3.97 \mathrm{mmol})$ in dry THF $(70 \mathrm{~mL})$ was added PCC (4.27 g, $19.8 \mathrm{mmol})$ and the resulting suspension allowed to stir at room temperature, under argon, overnight. The reaction was then diluted with $\mathrm{CH}_{2} \mathrm{Cl}_{2}$, and suction filtered to remove all solid material. The filtrate was concentrated and purified using column chromatography (10:3 hexanes/EtOAc) to afford 7 (0.99 $\mathrm{g}, 85 \%)$ as a colorless solid. All spectral data matched known compound. ${ }^{4}{ }^{1} \mathrm{H} \mathrm{NMR}\left(\mathrm{CDCl}_{3}\right) \delta$ 2.54 (s, 9H), $3.75(\mathrm{~s}, 9 \mathrm{H}) .{ }^{13} \mathrm{C} \mathrm{NMR}\left(\mathrm{CDCl}_{3}\right) \delta 32.5,64.4,127.1,155.3,200.6$.<smiles>CCc1c(C(C)=O)c(CC)c(C(C)=O)c(CC)c1C(C)=O</smiles> 
1,1',1'-(2,4,6-Triethylbenzene-1,3,5-triyl)triethanone (8). This compound was synthesized from a mixture of $\mathbf{6 a}$ and $\mathbf{6 b}(0.80 \mathrm{~g}, 2.7 \mathrm{mmol})$ and PCC $(2.93 \mathrm{~g}, 13.6 \mathrm{mmol})$ according to the same procedure reported for 7 to afford $8(0.745 \mathrm{~g}, 95 \%)$ as a colorless solid. All analytical data matched known compound. ${ }^{5}{ }^{1} \mathrm{H}$ NMR $\left(\mathrm{CDCl}_{3}\right) \delta 1.11(\mathrm{t}, J=7.5 \mathrm{~Hz}, 9 \mathrm{H}), 2.40(\mathrm{q}, J=7.5 \mathrm{~Hz}$, $6 \mathrm{H}), 2.53(\mathrm{~s}, 9 \mathrm{H}) .{ }^{13} \mathrm{C} \mathrm{NMR}\left(\mathrm{CDCl}_{3}\right) \delta 16.5,24.3,33.1,134.1,141.4,206.8$.<smiles>COc1c(O)c(OC)c(C(C)O)c(OC)c1C(=O)Cc1c(OC)c(C(C)O)c(OC)c(C(C)O)c1OC</smiles>

meso-3-(1-Hydroxyethyl)-5-(1-hydroxyethyl)-2,4,6-trimethoxybenzaldehyde (meso-9) and ( \pm )-3-(1-hydroxyethyl)-5-(1-hydroxyethyl)-2,4,6-trimethoxybenzaldehyde $(( \pm)-9)$. To a stirring solution of methyllithium $(1.6 \mathrm{M}$ in ether, $3.0 \mathrm{mmol})$ in dry THF $(15 \mathrm{~mL})$ at $-78^{\circ} \mathrm{C}$ under a blanket of argon, was slowly added a solution of $1(0.15 \mathrm{~g}, 0.60 \mathrm{mmol}$, dissolved in $5 \mathrm{~mL}$ dry THF) via syringe. The resulting mixture was maintained at $-78{ }^{\circ} \mathrm{C}$ for an additional hour, and then allowed to gradually warm to $0{ }^{\circ} \mathrm{C}$ over $2 \mathrm{~h}$. The reaction was then quenched by dropwise addition of dilute $\mathrm{HCl}$, and extracted with EtOAc. The combined organic extracts were dried over $\mathrm{MgSO}_{4}$, filtered, and concentrated to a crude residue which was purified via flash chromatography (4:1 EtOAc/hexanes) to afford 5a $(0.09 \mathrm{~g}, 53 \%)$ as a colorless oil, and a 1:1 mixture of meso-9:( \pm -9 $(0.047 \mathrm{~g}, 28 \%)$ as a colorless oil: IR (film): $v_{\max } 3428,2941,1687$, 1572, $1131 \mathrm{~cm}^{-1} ;{ }^{1} \mathrm{H}$ NMR $\left(\mathrm{CDCl}_{3}\right) \delta 1.58(\mathrm{~m}, 12 \mathrm{H}), 3.32(\mathrm{bs}, 4 \mathrm{H}), 3.85(\mathrm{~s}, 3 \mathrm{H}), 3.88(\mathrm{~s}, 3 \mathrm{H})$, $3.93(\mathrm{~s}, 6 \mathrm{H}), 3.94(\mathrm{~s}, 6 \mathrm{H}), 5.12$ (quin, $J=6.9 \mathrm{~Hz}, 4 \mathrm{H}), 10.22(\mathrm{~s}, 1 \mathrm{H}), 10.23(\mathrm{~s}, 1 \mathrm{H}) .{ }^{13} \mathrm{C} \mathrm{NMR}$ $\left(\mathrm{CDCl}_{3}\right) \delta 24.3,24.5,63.8,64.0,64.2,65.1,128.4,128.5,161.8,187.5,187.7$. HRMS (ESI) calcd for $\mathrm{C}_{14} \mathrm{H}_{20} \mathrm{O}_{6}(\mathrm{M}+\mathrm{Na})^{+} 307.1152$, found 307.1164 . 


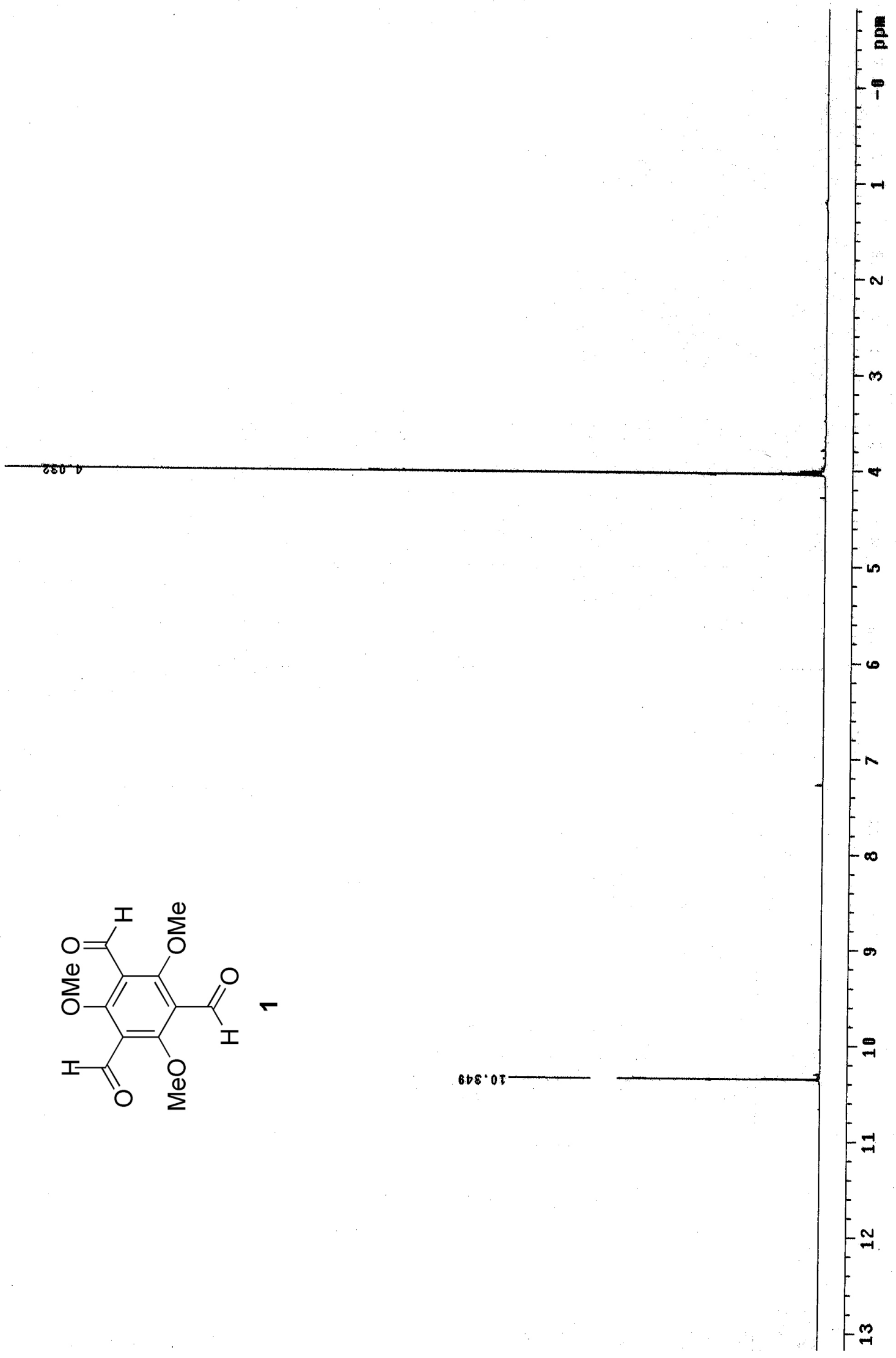




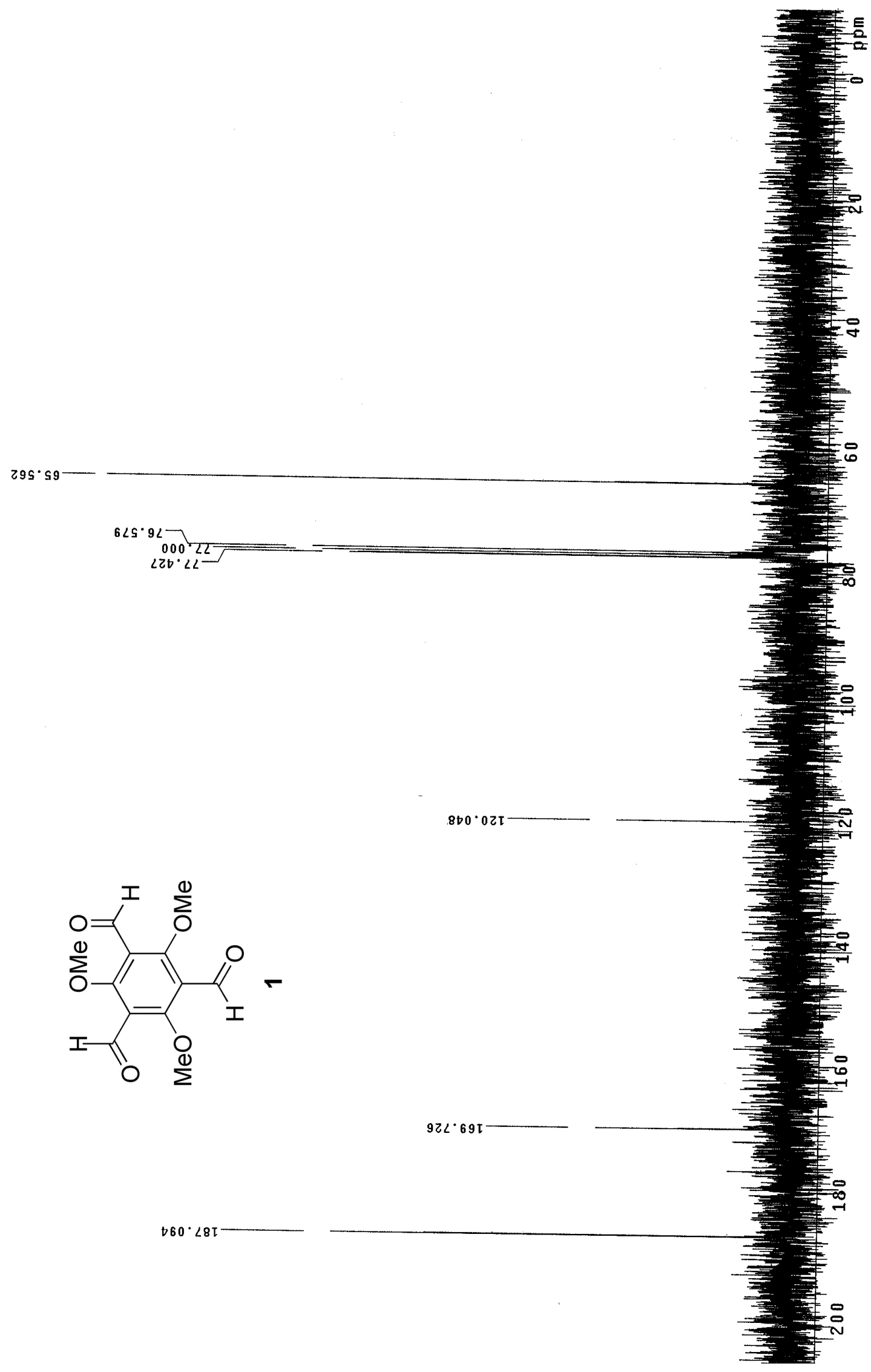




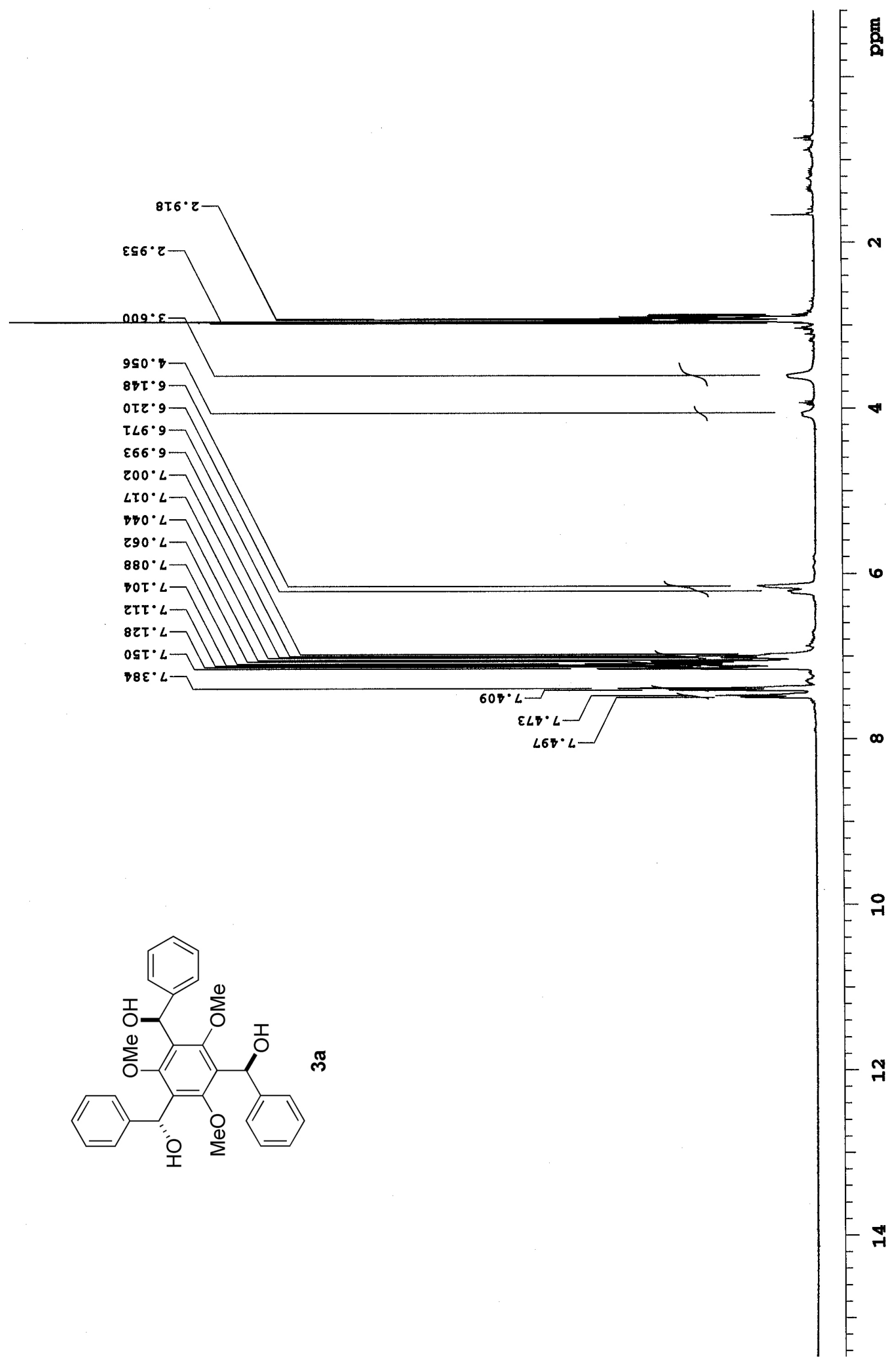




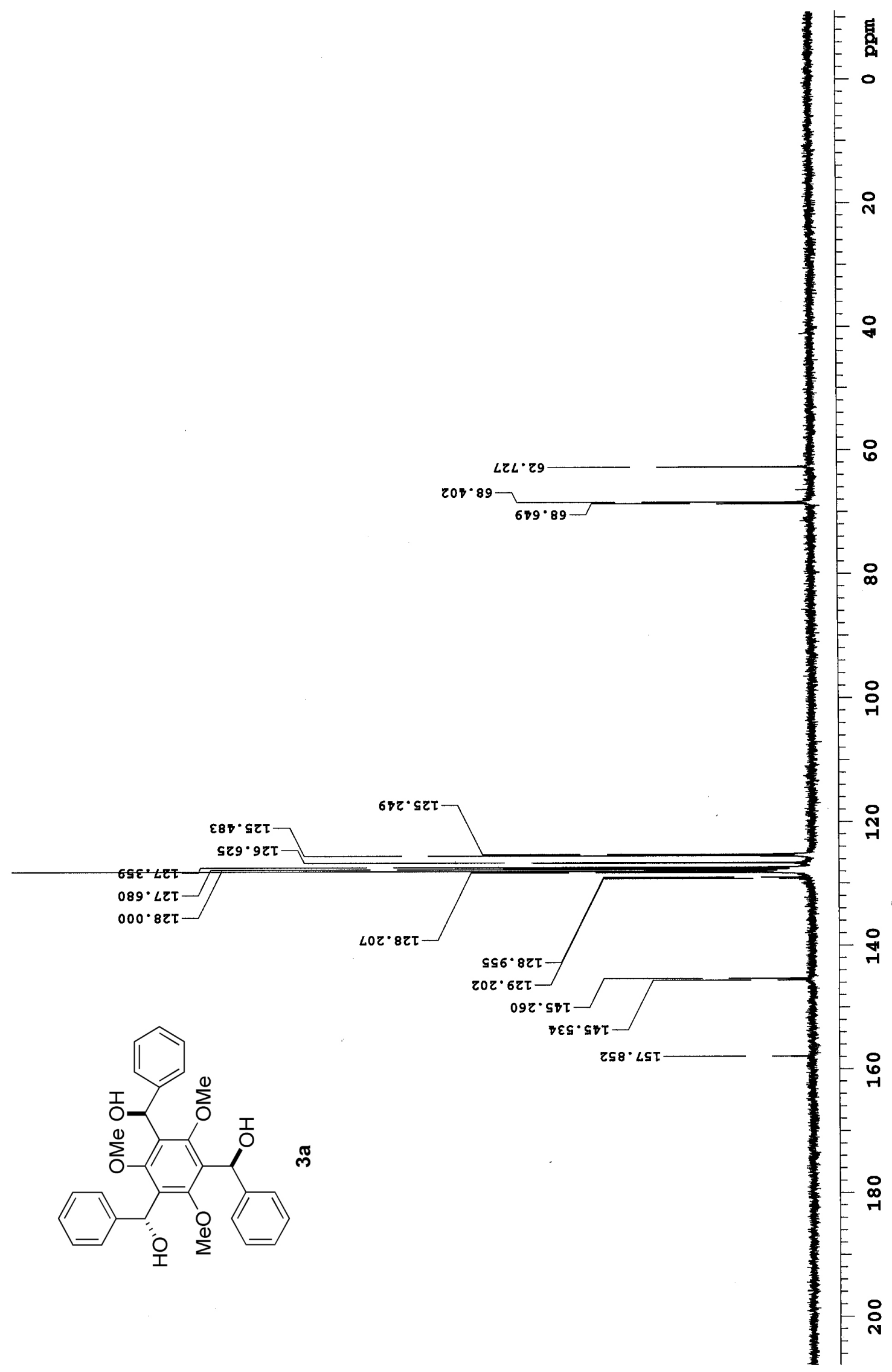



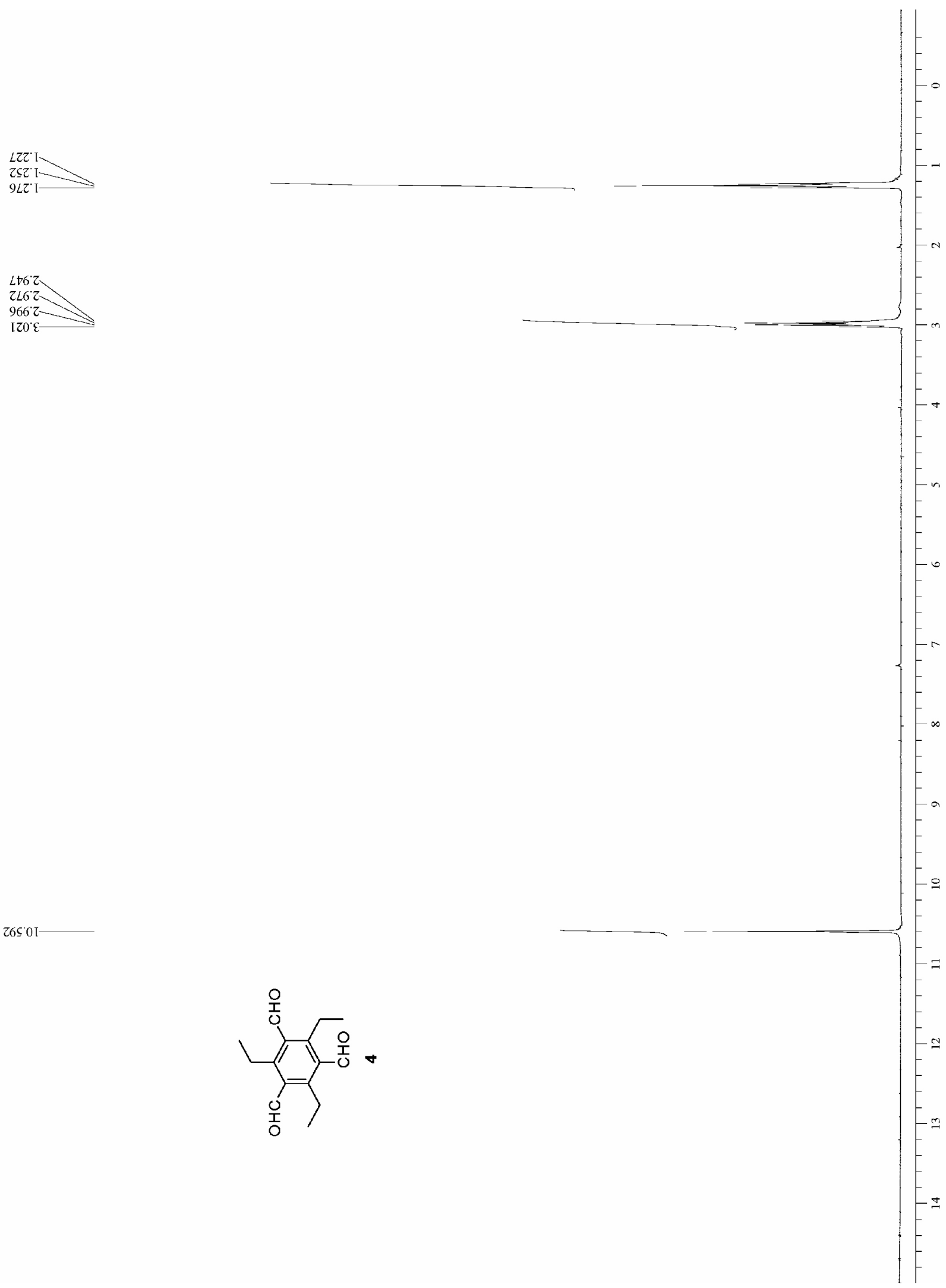


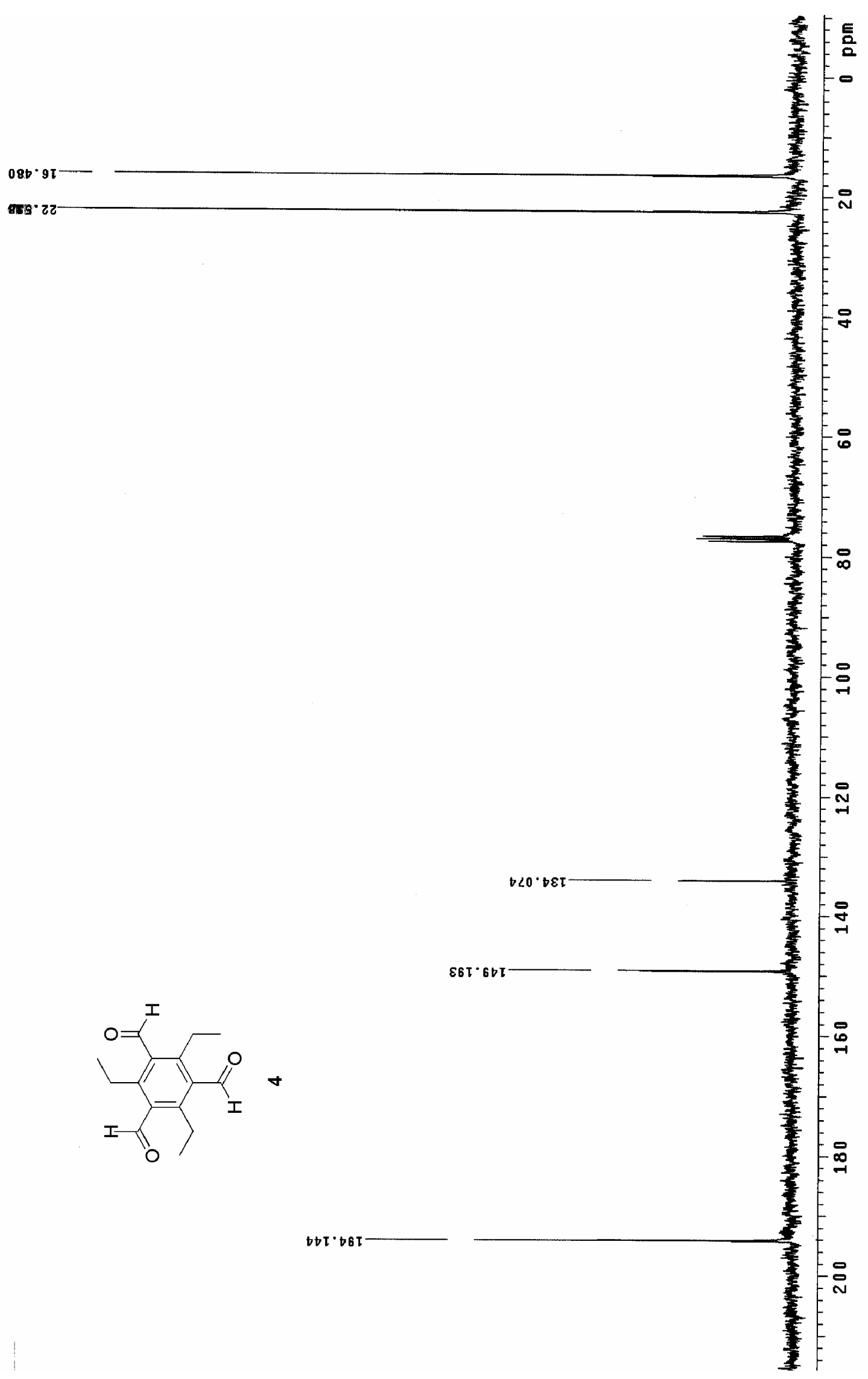



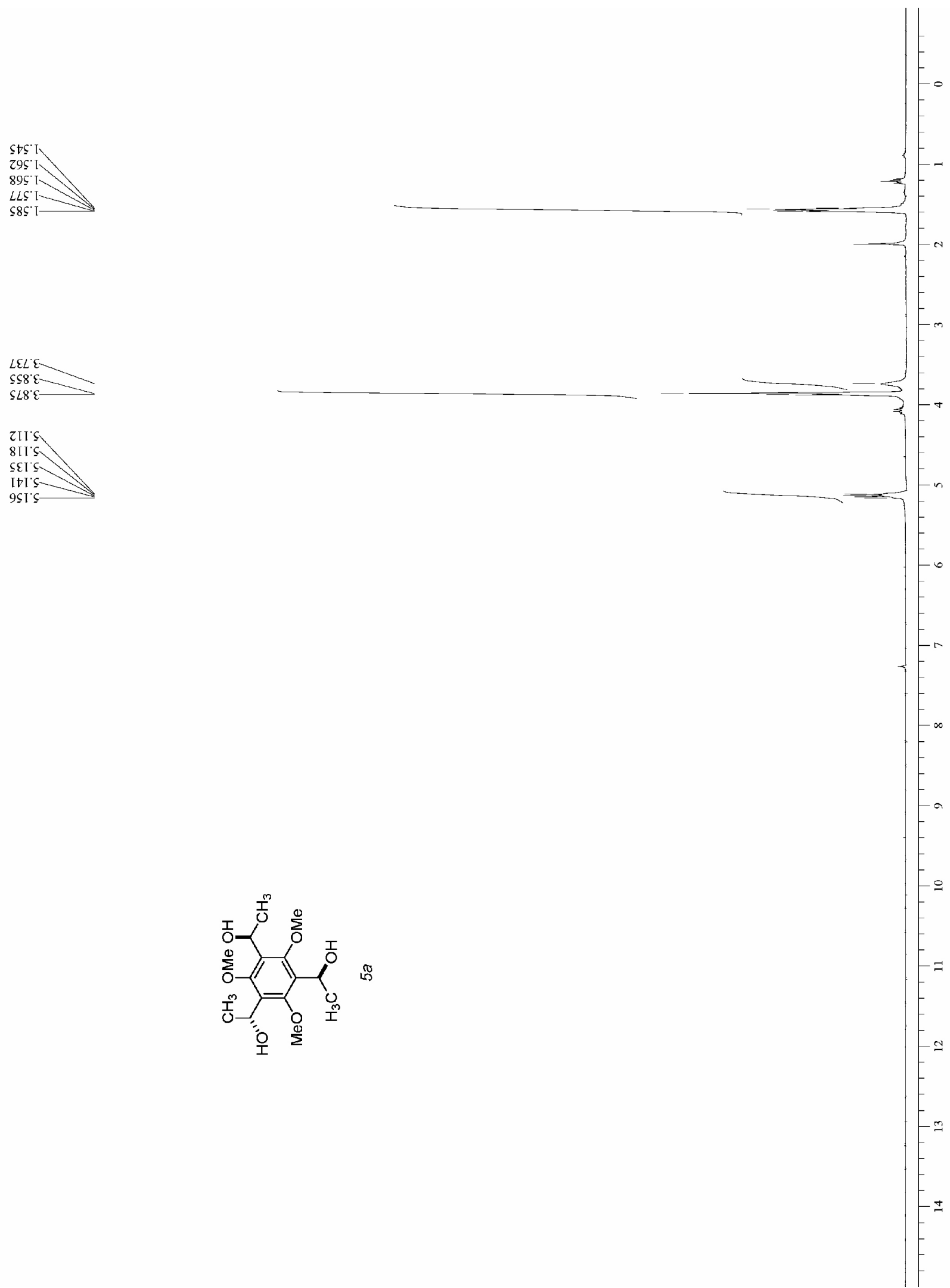

zII'S

SII $\varsigma$ I $\varsigma$

ItI's

$9 \varsigma I^{\prime} \varsigma$ 

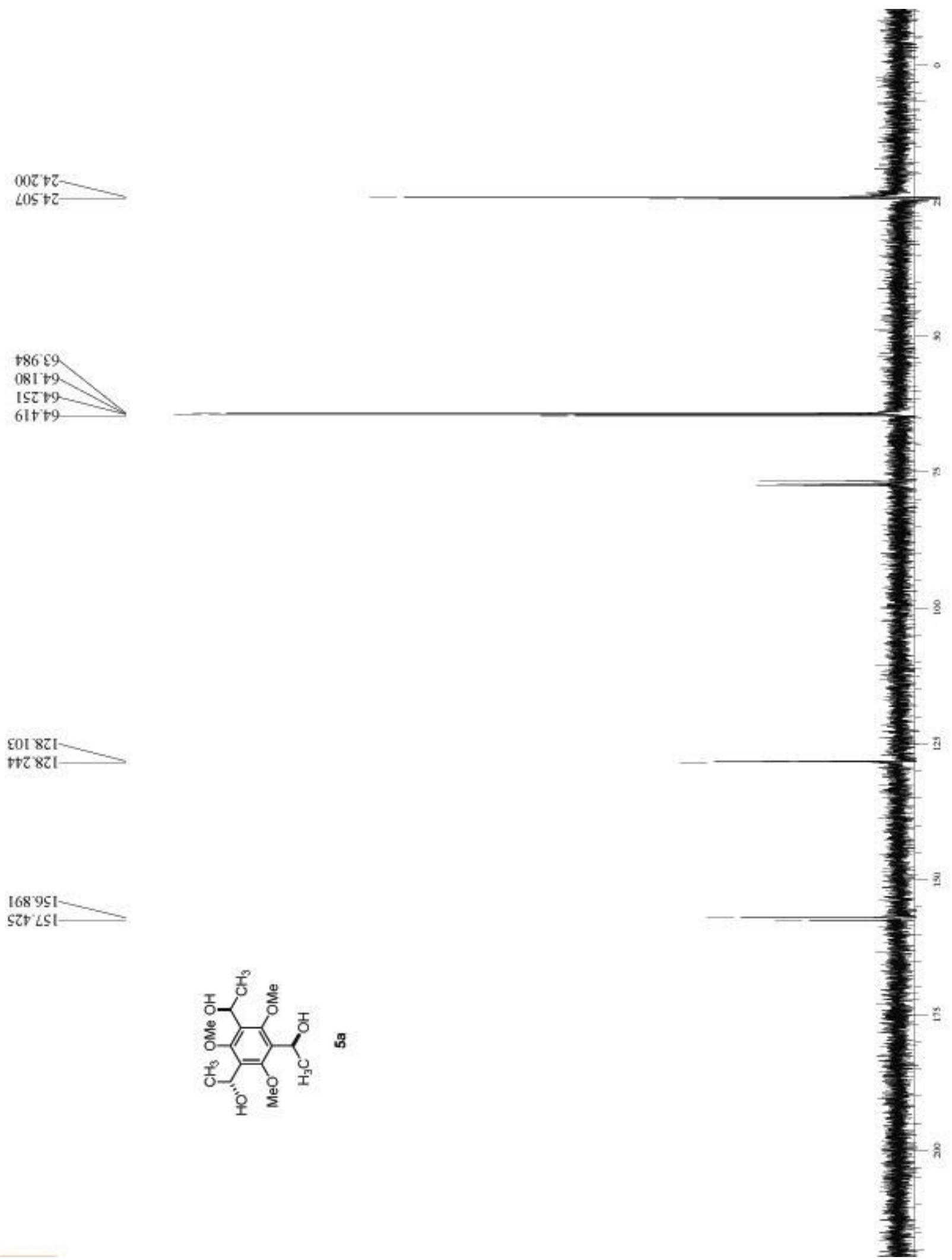


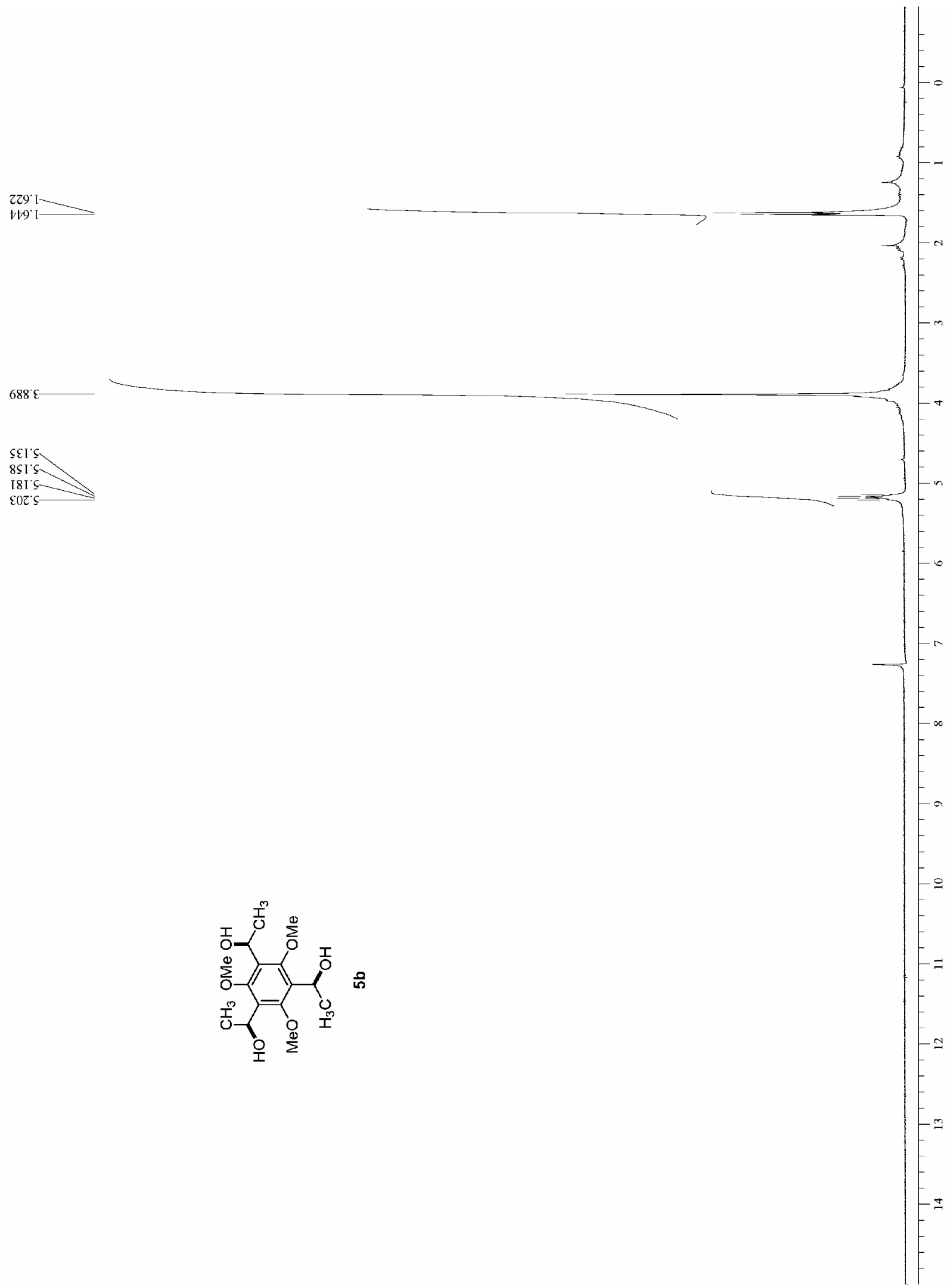




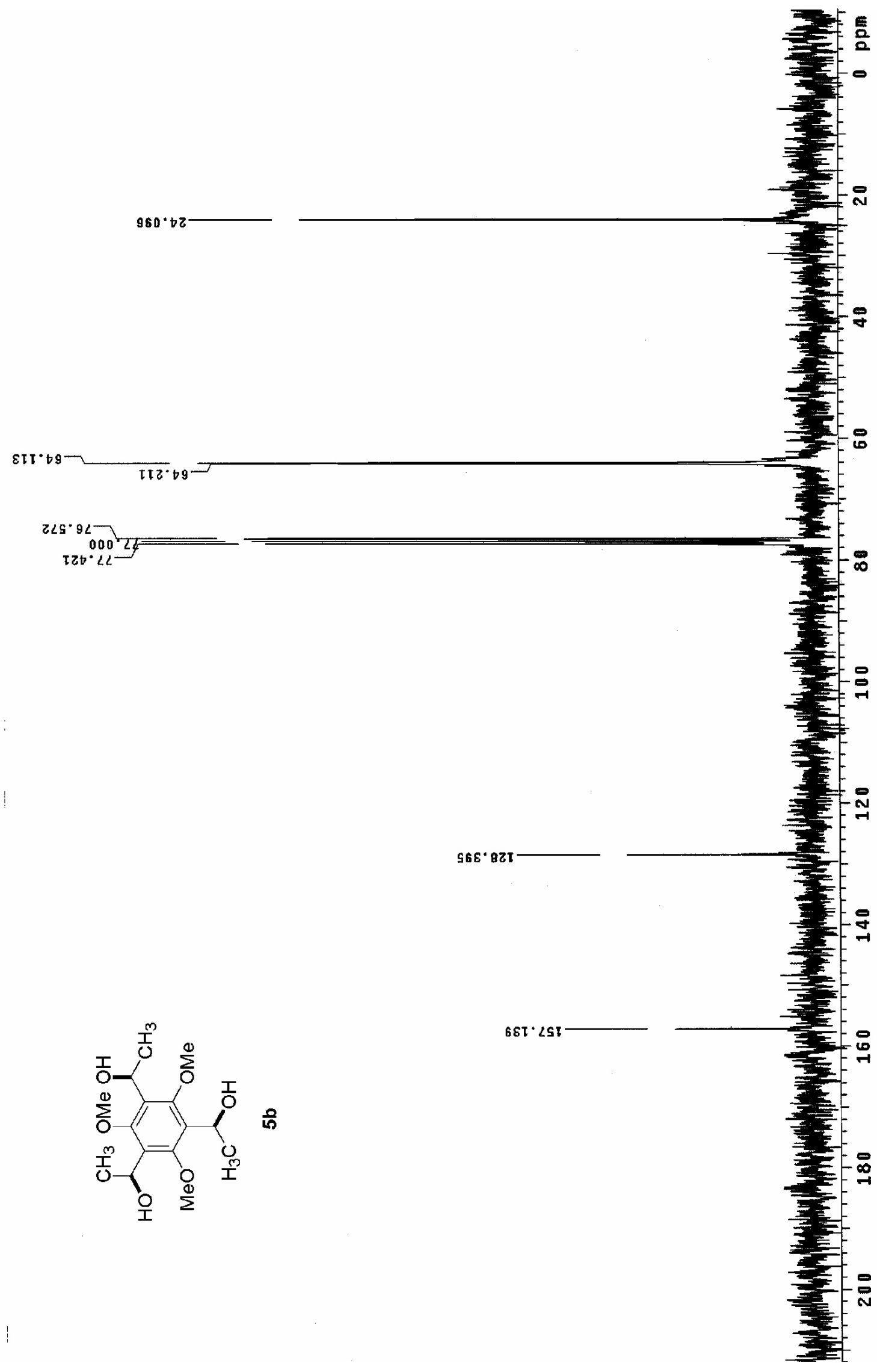



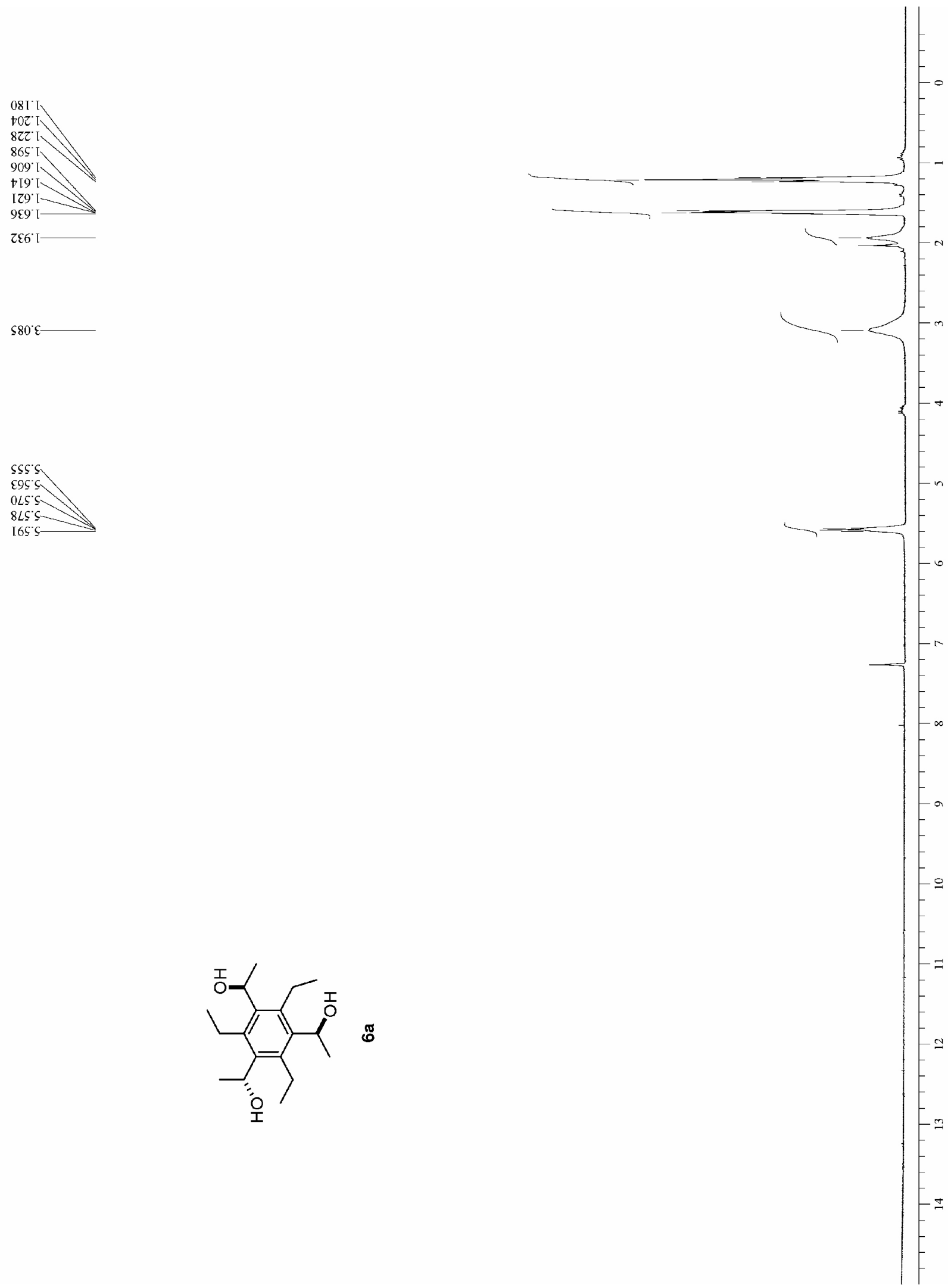
$9 \varepsilon \varepsilon \angle 1$

$08 \varepsilon L I-$

ILSZZ

ctog co

$\angle \& L E Z \cdots$

0₹999

51299

$2666 \varepsilon 1$

$\left|81^{\circ}\right| t \mid$

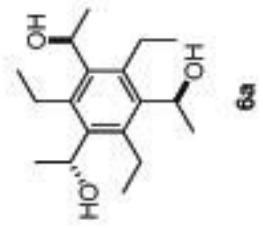

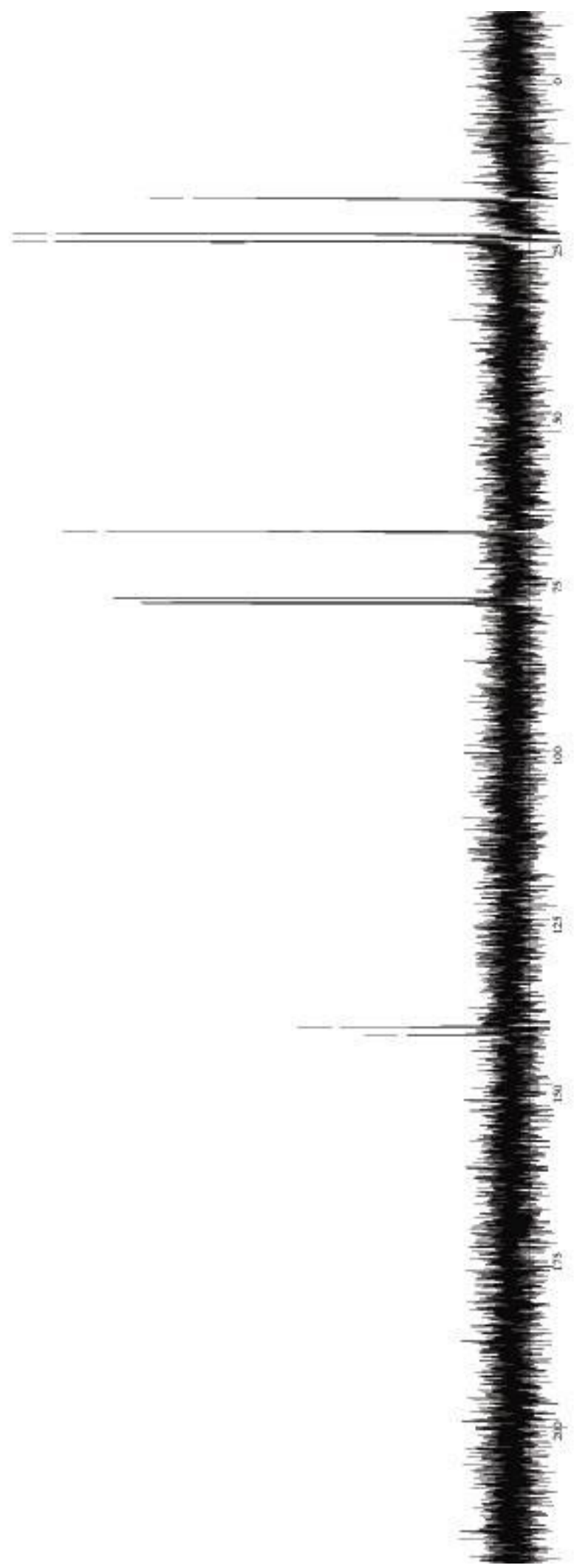



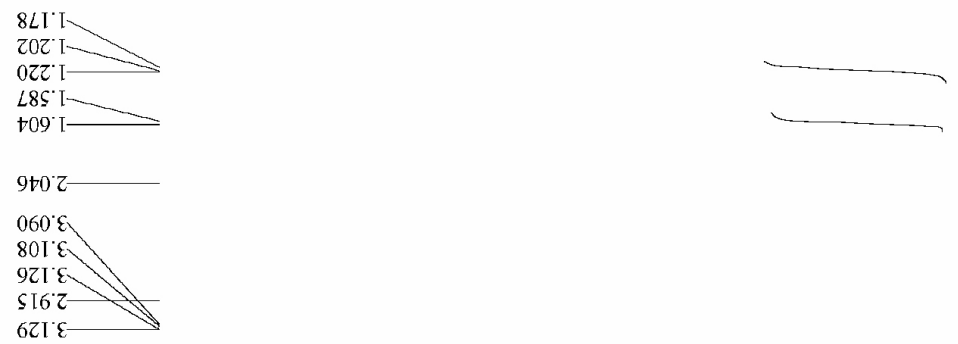

SIS

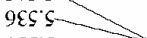

LSS
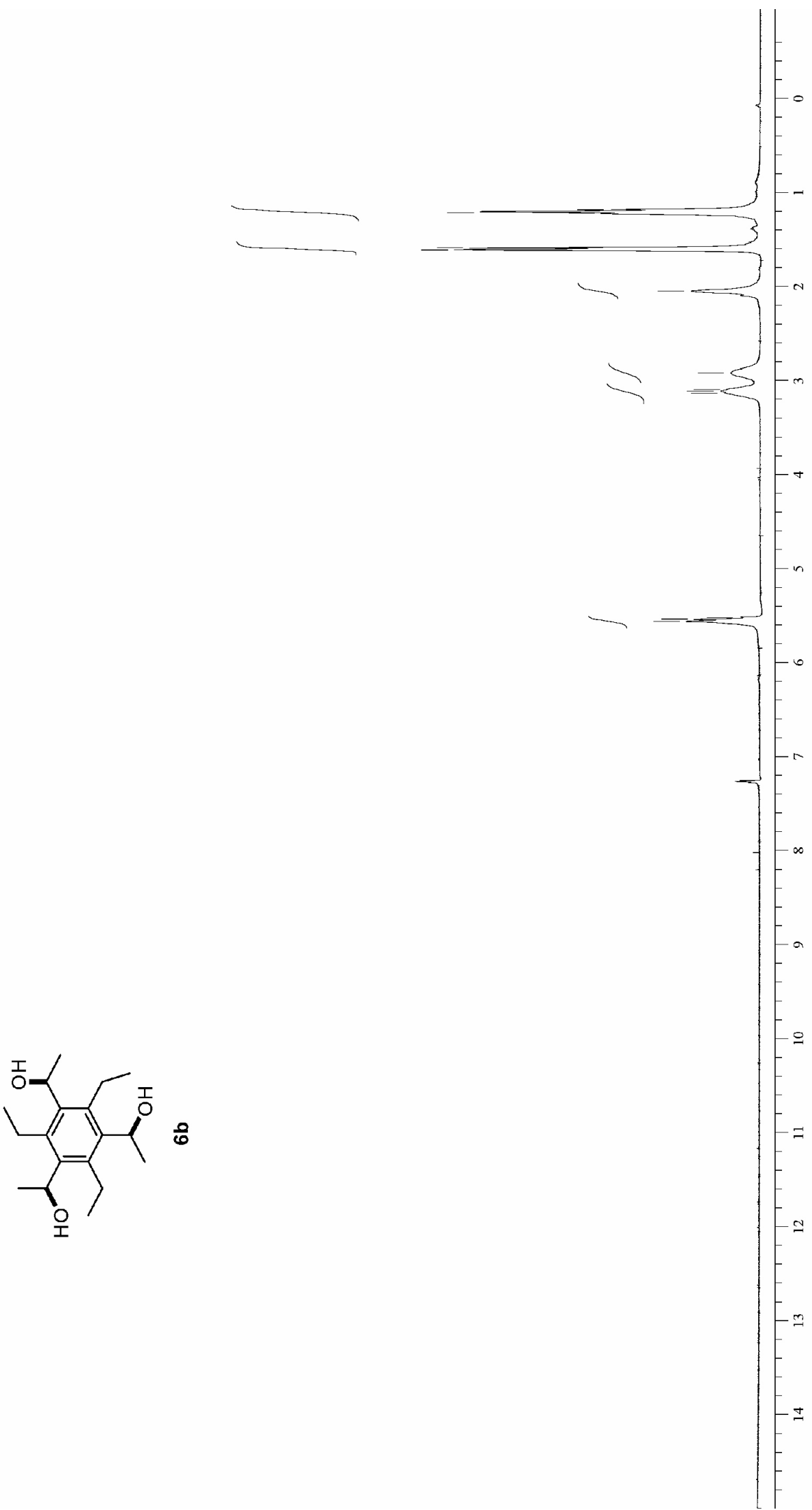


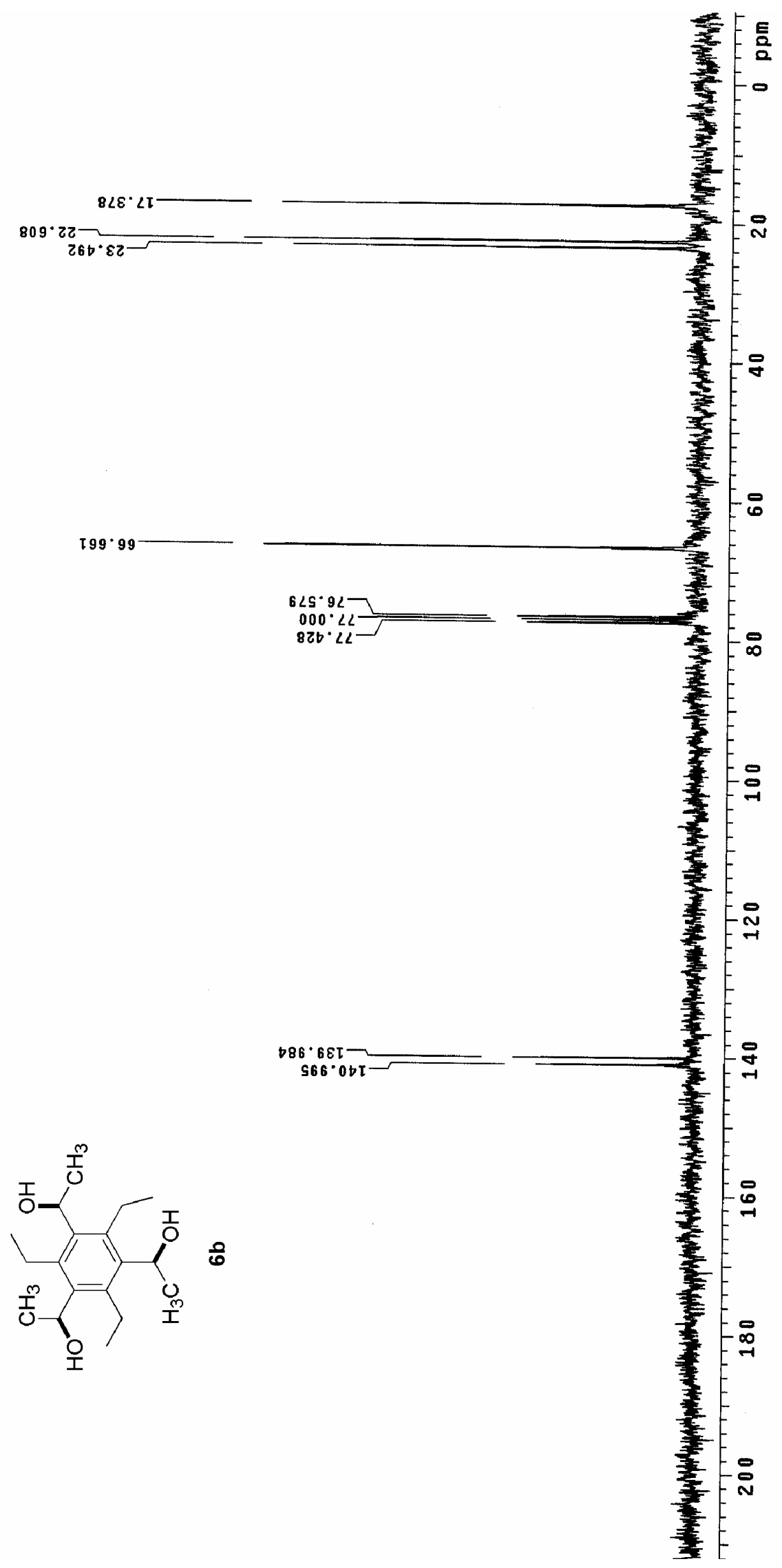




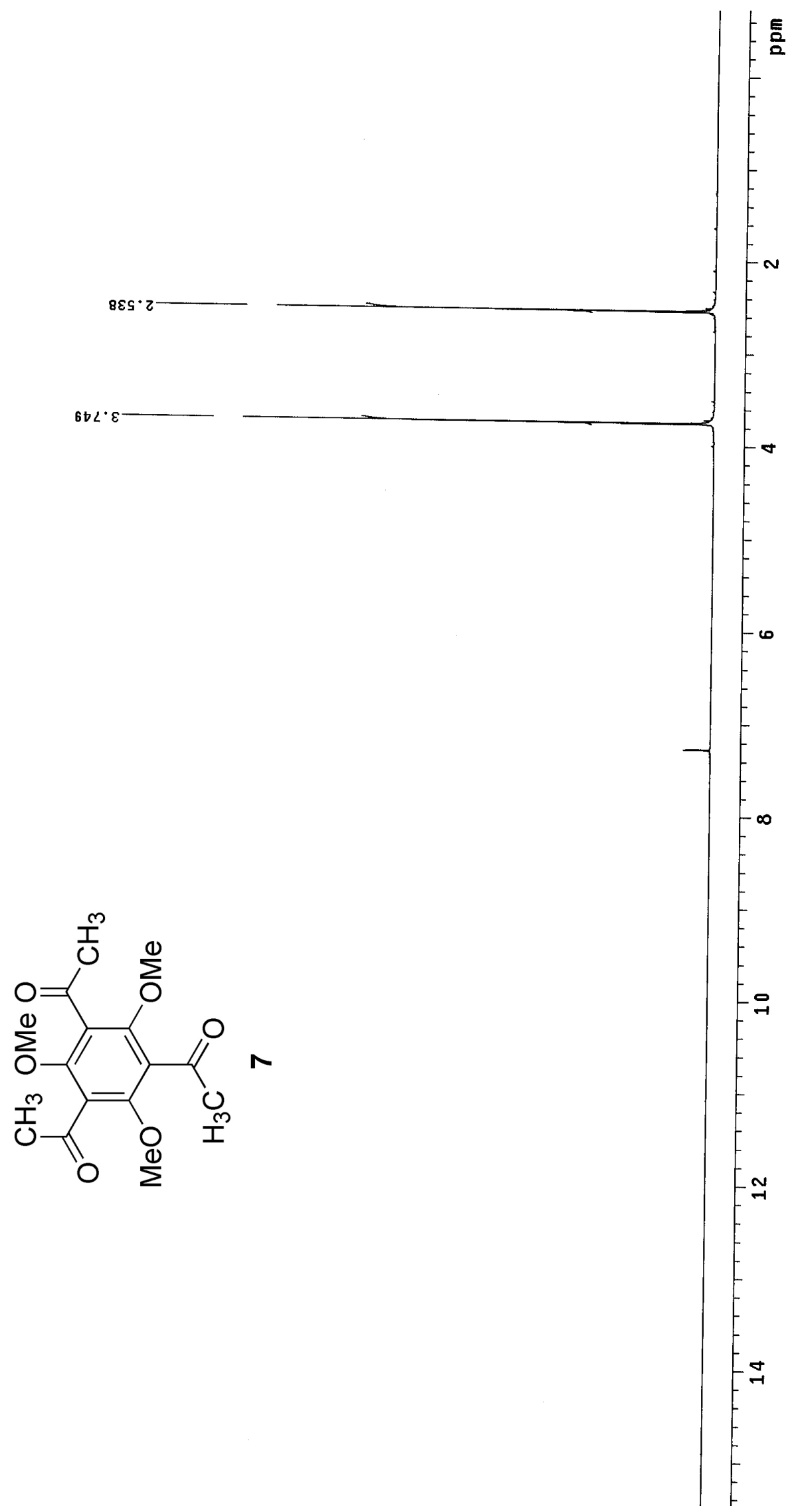




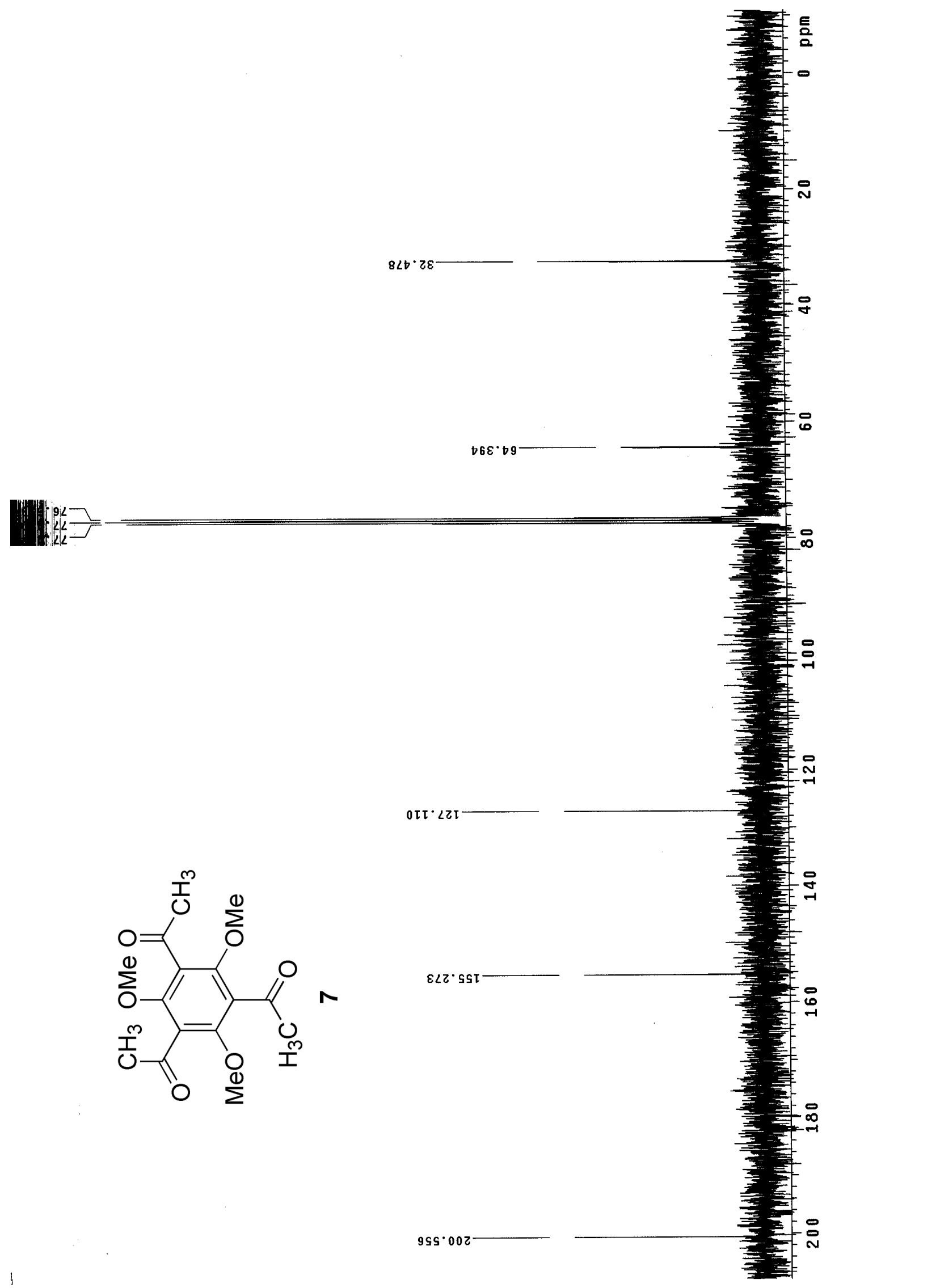




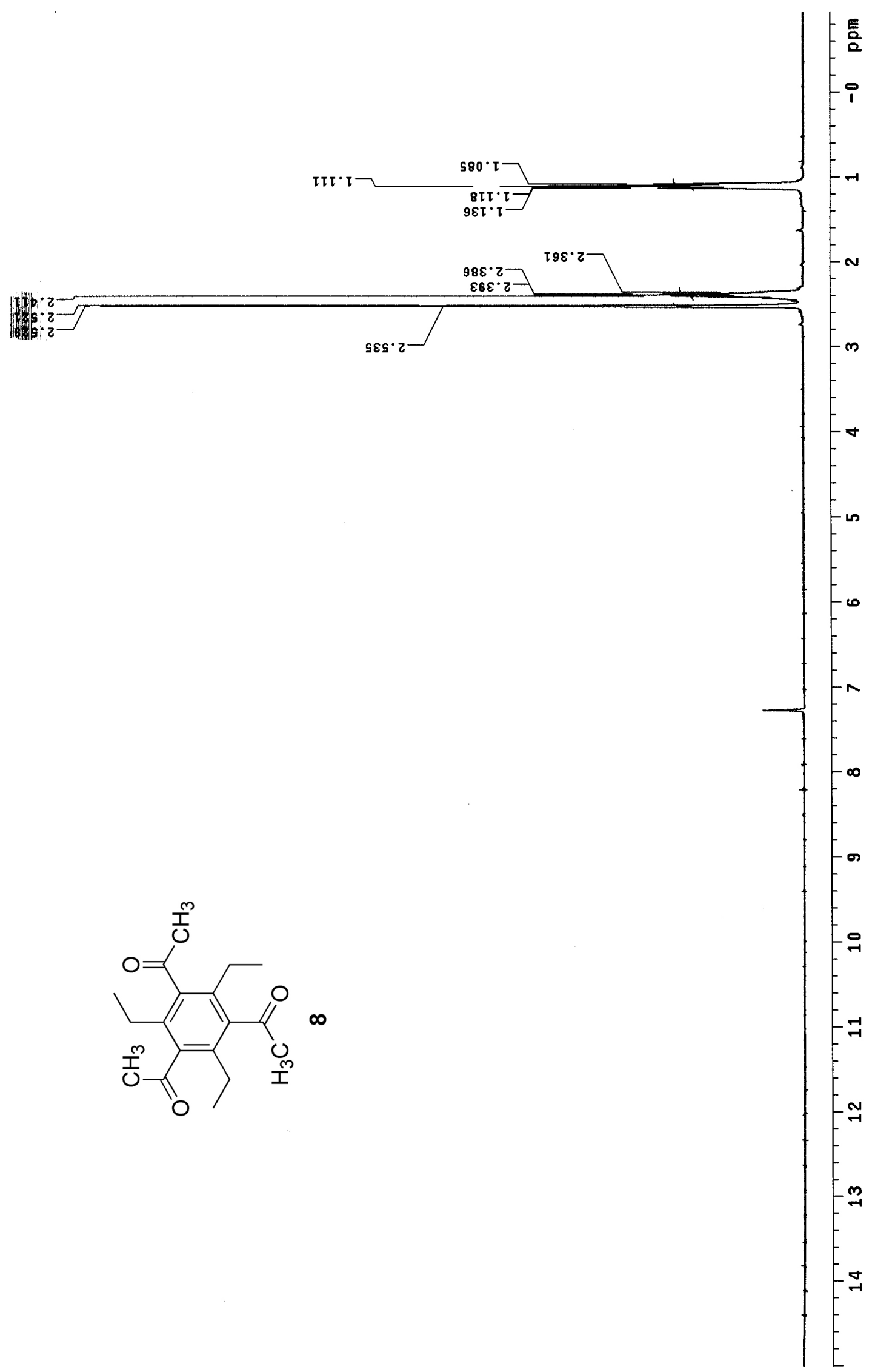




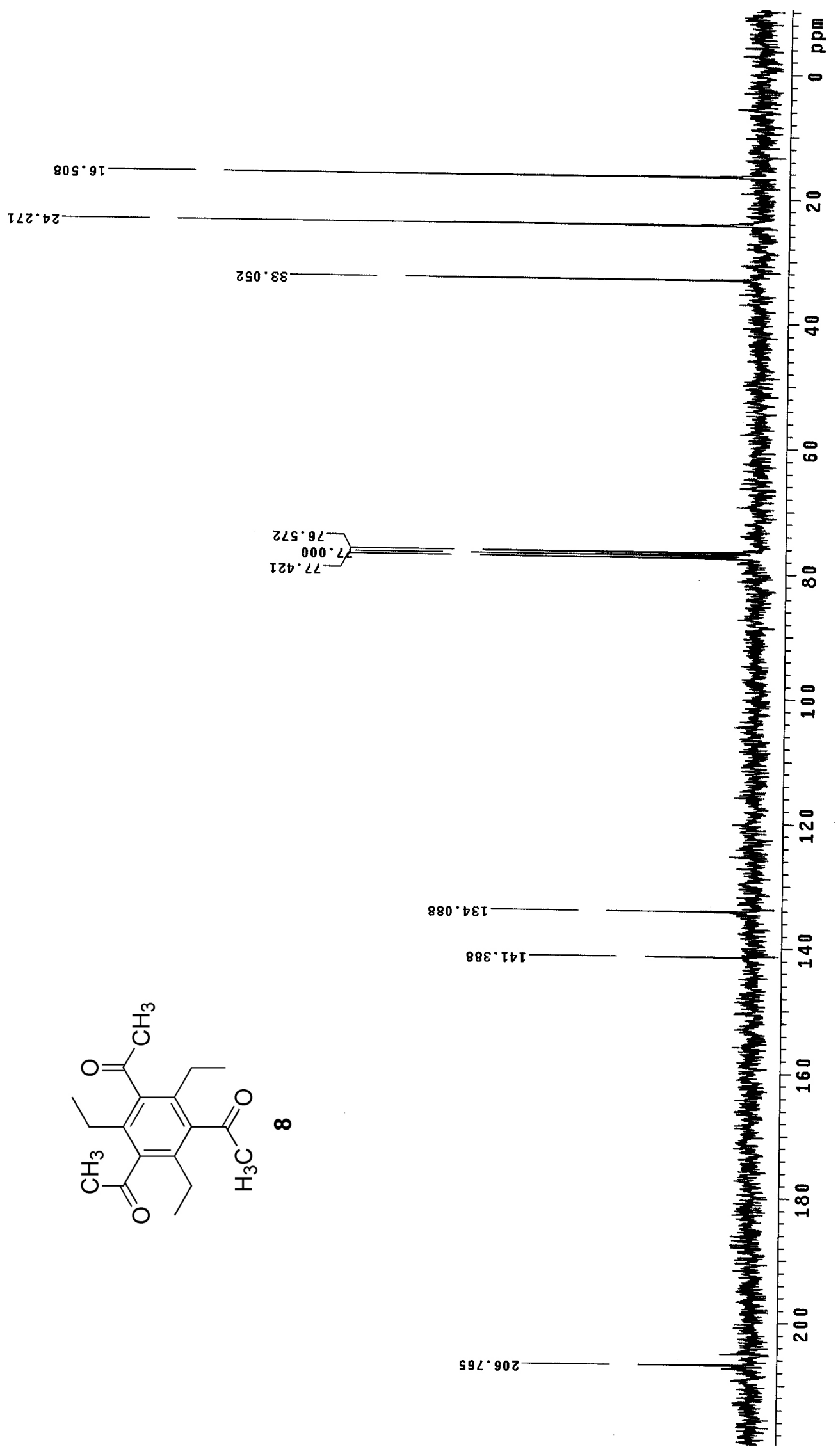


$6 \varsigma^{\circ} \mathrm{I}$

$285^{\circ} \mathrm{I}-\mathrm{2}$

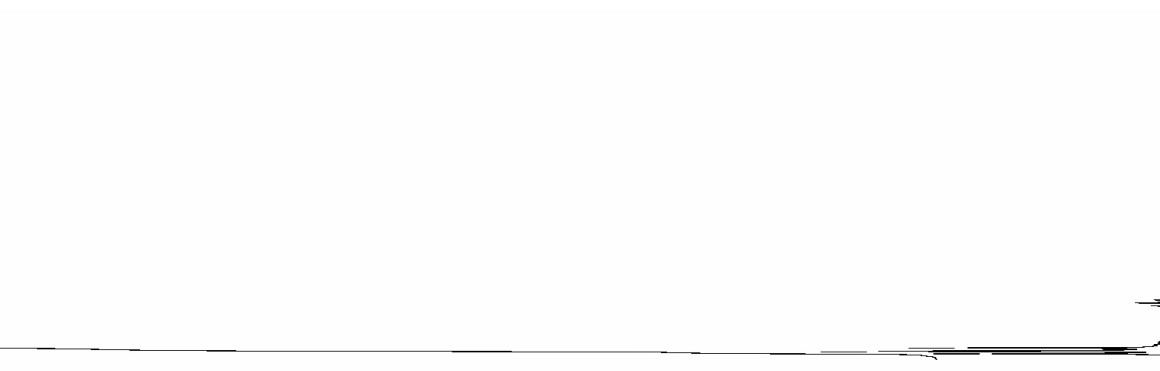

$2 \varsigma 8^{\circ} \varepsilon$

$\subseteq \angle 8^{\circ} \mathrm{E}$

$0 \mathcal{E} 6 \mathcal{E}$

Et6

8LI'S

$\angle 80^{\circ} \subseteq$

$60 \mathrm{I}^{\circ} \mathrm{S}$

zEIS

SSI $\mathrm{L}$
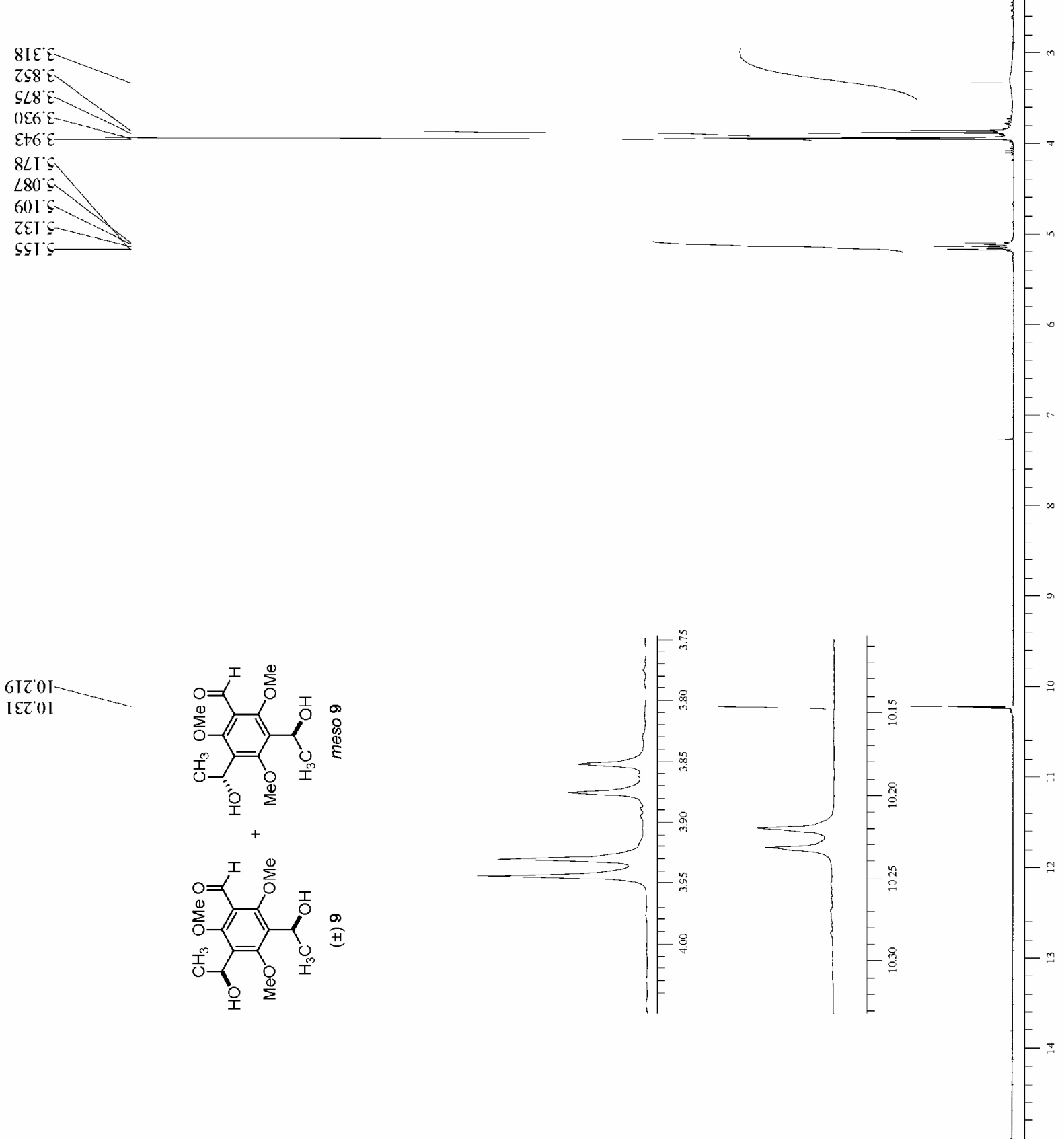


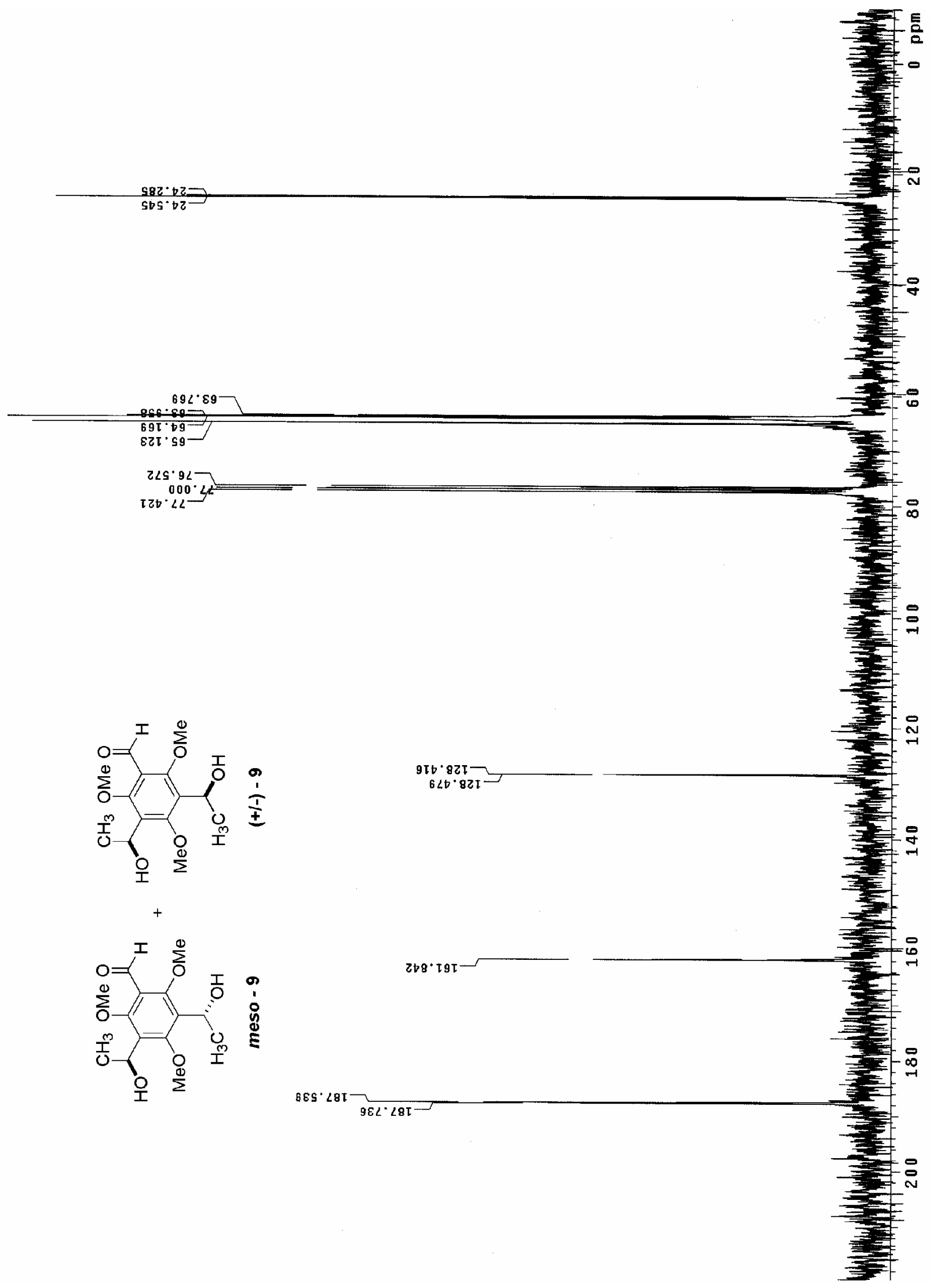


1. Domagalska, B. W.; Syper, L.; Wilk, K. A. Tetrahedron 2004, 60, 1931-1939.

2. Simaan, S.; Siegel, J. S.; Biali, S. E. J. Org. Chem. 2003, 68, 3699-3701.

3. Kilway, K. V.; Siegel, J. S. Tetrahedron 2001, 57, 3615-3627.

4. Dean, F. M.; Robertson, A. J. Chem. Soc. 1953, 1241-1249.

5. Reimschneider, R. Z. Naturforsch., B: Chem. Sci. 1956, 291-301. 